\title{
XXXIII. Simplified deduction of the formula from the theory of combinations which Planck uses as the basis of his radiation theory
}

\section{P. Ehrenfest \& H. Kamerlingh Onnes}

To cite this article: P. Ehrenfest \& H. Kamerlingh Onnes (1915) XXXIII. Simplified deduction of the formula from the theory of combinations which Planck uses as the basis of his radiation theory, Philosophical Magazine Series 6, 29:170, 297-301, DOI: 10.1080/14786440208635308

To link to this article: http://dx.doi.org/10.1080/14786440208635308

冓 Published online: 08 Apr 2009.

Submit your article to this journal $[\pi$

Џll Article views: 7

Q View related articles $\longleftarrow$

Citing articles: 1 View citing articles $\square$ 
series spectrum of hydrogen consists only of lines which are represented by the formula

$$
\nu=109704\left(\frac{1}{n_{1}^{2}}-\frac{1}{n_{2}^{2}}\right),
$$

and that the series spectrum of helium in addition to the ordinary helium lines consists of all the lines which are represented by the formula

$$
\nu=109750\left\{\frac{1}{\left(\frac{n_{1}}{2}\right)^{2}}-\frac{1}{\left(\frac{n_{2}}{2}\right)^{2}}\right\} .
$$

The author wishes to thank Sir Ernest Rutherford for bringing the subject of the present investigation to his notice and for valuable snggestions and encouragement during the course of the research.

The University of Manchester, Dec. 1914.

XXXIII. Simplified Deduction of the Formula from the Theory of Combinations which Planck uses as the Basis of his Radiation Theory. By P. Ehrenfest and H. KamerLINGH ONNEs*

WE refer to the expression

$$
\mathrm{r}_{\mathrm{P}}^{\mathrm{N}}=\frac{(\mathrm{N}-1+\mathrm{P}) !}{\mathrm{P} !(\mathrm{N}-1) !}, \quad \text {. . . . . }
$$

which gives the number of ways in which $\mathrm{N}$ monochromatic resonators $R_{1}, R_{2}, \ldots R_{N}$ may be distributed over the various degrees of energy, determined by the series of multiples $0, \epsilon, 2 \epsilon \ldots$ of the unit energy $\epsilon$, when the resonators together must each time contain the given multiple $\mathrm{P}_{\epsilon}$. Two methods of distribution will be called identical, and only then, when the first resonator in the one distribution is at, the same grade of energy as the same resonator in the second, and similarly the second, third,.... and the Nth resonator are each at the same energy-grades in the two distributions.

Taking a special example, we shall introduce a symbol for the distribution. Let $N=4$, and $P=7$. One of the possible distributions is the following : resonator $R_{1}$ has reached the

* Communicated by the Authors. 


\section{Profs. P. Ehrenfest and H. K. Onnes : Simplified}

energy-grade $4 \epsilon\left(R_{1}\right.$ contains the energy $\left.4 \epsilon\right), R_{2}$ the grade $2 \epsilon, R_{3}$ the grade $0 \epsilon$ (contains no energy), $R_{4}$ the grade $\epsilon$. Our symbol will, read from left to right, indicate the energy of $R_{1}, R_{2}, R_{3}, R_{4}$, in the distribution chosen, and particularly express that the total energy is $7 \varepsilon$. For this case the symbol will be :-

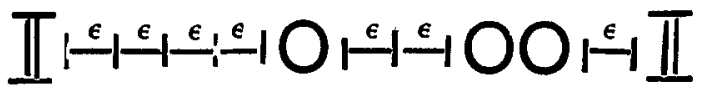

or also more simply :--

$$
\text { II } \epsilon \epsilon \epsilon \in \mathrm{O} \epsilon \epsilon \mathrm{O} \mathrm{O} \epsilon
$$

With general values of $\mathrm{N}$ and $\mathrm{P}$ the symbol will contain $P$ times the sign $\epsilon$ and $(N-1)$ times the sign $\mathbf{O}^{*}$. The question now is, how many different symbols for the distribution may be formed in the manner indicated above from the given number of $\epsilon$ and $O$ ? The answer is

$$
\frac{(\mathrm{N}-1+\mathrm{P}) !}{\mathrm{P} !(\mathrm{N}-1) !} \cdot \text {. . . . . }
$$

Proof : first considering the $(\mathrm{N}-1+\mathrm{P})$ elements $\epsilon \ldots \epsilon$, $O \ldots O$ as so many distinguishable entities, they may be arranged in

$$
(\mathrm{N}-1+\mathrm{P}) \text { ! }
$$

different manners between the ends II II. Next note, that each time

$$
(\mathrm{N}-1) ! \mathrm{P}: \text {. . . . . . }
$$

of the combinations thus obtained give the same symbol for the distribution (and give the same energy-grade to each resonator), viz. all those combinations which are formed from each other by the permutation of the $P$ elements $\epsilon$ the $(\mathrm{N}-1)$ elements $\mathrm{O}$. The number of the different symbols for the distribution and that of the distributions themselves required is thus obtained by dividing (2) by (3) q.e.d.

* We were led to the introduction of the $(\mathrm{N}-1)$ partitions between the $N$ resonators in trying to find an explanation of the form $(N-1)$ ! in the denominator of $(A)$. Planck proves that the number of distributions mnst be equal to the number of all "combinations with repetitions of $\mathrm{N}$ elements of class $\mathrm{P}$," and for the proof, that this number is given by the expression (A), he refers to the train of reasoning followed in treatises on combinations for this particular case. In these treatises the expression (A) is arrived at by the aid of the device of "transition from $n$ to $n+1$," and this method taken as a whole does not give an insight into the origin of the final expression.

+ See Appendix. 


\section{APPENDIX.}

The contrasl between Planck's hypothesis of the energy-grades and Einstein's hypothesis of energy-quanta.

The permutation of the elements $\epsilon$ is a purely formal device, just as the permutation of the elements $O$ is. More than once the analogous, equaliy formal device used by Planck, viz. distribution of $P$ energy-elements over $N$ resonators, has by a misunderstanding been given a physical interpretation, which is absolutely in conflict with Planck's radiation-formula and would lead to Wien's radiationformula.

As a matter of fact, Planck's energy-elements were in that case almost entirely identified with Einstein's lightquanta, and accordingly it was said that the difference between Planck and Einstein consists herein, that the latter assumes the existence of mutually independent energyquanta also in empty space, the former only in the interior of matter, in the resonators. The confusion which underlies this view has been more than once pointed out*. Einstein really considers $\mathrm{P}$ similar quanta, existing independently of each other. He discusses, for instance, the case that they distribute themselves irreversibly from a space of $\mathrm{N}_{1} \mathrm{~cm}^{3}$ over a larger space of $\mathrm{N}_{2} \mathrm{~cm}^{3}$, and he finds, using Boltzmann's entropy-formula, $\mathrm{S}=k \log W$, that this produces a gain of entropy $\dagger$ :

$$
\mathrm{S}-\mathrm{S}_{0}=k \log \left(\frac{\mathbf{N}_{2}}{\mathbf{N}_{1}}\right)^{\mathbf{P}}, \ldots \cdot \cdot \cdot \cdot
$$

i.e. the same increase as in the analogous irreversible distribution of $P$ similar independent gas-molecules, for the number of ways in which $P$ quanta may be distributed, first over $N_{1}$, then over $N_{2}$ cells in space, are to each other in the ratio

$$
\mathrm{N}_{1}^{\mathrm{P}}: \mathrm{N}_{2}^{\mathrm{P}} \text {. . . . . . . }
$$

If with Planck the object were to distribute $\mathrm{P}$ mutually independent elements $\epsilon$ over $\mathrm{N}$ resonators, in passing from $N_{1}$ to $N_{2}$ resonators the number of possible distributions would in this case also increase in the ratio $(\beta)$ and correspondingly the entropy according to equation $(\alpha)$. We know,

* P. Ehrenfest, Ann. d. Phys. vol. xxxvi. p. 91 (1911); G. Krutkow, Physik. Zselk. vol. xv. pp. 133, 363 (1914).

† A. Einstein, Ann. d. Phys. vol. xvii. p. 132 (1905). 
however, that Planck obtains the totally different formula

$$
\frac{\left(\mathrm{N}_{1}-1+\mathrm{P}\right) !}{\left(\mathrm{N}_{1}-1\right) ! \mathrm{P} !}: \frac{\left(\mathrm{N}_{2}-1+\mathrm{P}\right) !}{\left(\mathrm{N}_{2}-1\right) ! \mathrm{P} !} \cdot \cdot \cdot(\gamma)
$$

(which only corresponds approximately with $(\beta)$ for very large values of $P$ ) and a corresponding law of dependence of the entropy on $N$. This can be simply explained as follows: Planck does not deal with really mutually free quanta $\epsilon$; the resolntion of the multiples of $\epsilon$ in separate elements $\epsilon$, which is essential in his method, and the introduetion of these separate elements have to be taken cum grano salis; it is simply a formal device entirely analogous to our permutation of the elements $\epsilon$ or $O$. The real object which is counted remains the number of all the different distributions of $\mathrm{N}$ resonators over the energy-grades $0, \epsilon$, $2 \epsilon .$. with a given total energy $\mathrm{P} \epsilon$. If, for instance, $\mathrm{P}=\mathbf{3}$, and $\mathrm{N}=2$, Einstein has to distinguish $2^{3}=8$ ways in which the three (similar) light-quanta $\mathrm{A}, \mathrm{B}, \mathrm{C}$ can be distributed over the space-cells 1,2 .

\begin{tabular}{r|ccc} 
& A. & B. & C. \\
\hline I. & 1. & 1 & 1 \\
II. & 1 & 1 & 2 \\
III. & 1 & 2 & 1 \\
IV. & 1 & 2 & 2 \\
V. & 2 & 1 & 1 \\
VI. & 2 & 1 & 2 \\
VII. & 2 & 2 & 1 \\
VIII. & 2 & 2 & 2
\end{tabular}

Planck, on the. other hand, must count the three cases II., III., and V. as a single one, for all three express that resonator $R_{1}$ is at the grade $2 \epsilon, R_{2}$ at $\epsilon$; similarly, he has to reckon the cases IV., VI., and VII. as one; $R_{1}$ has here $\epsilon$ and $R_{2} 2 e$. Adding the two remaining cases $I$. $\left(R_{1}\right.$ contains $\left.3 \epsilon, \mathrm{R}_{2} 0 \epsilon\right)$ and II. ( $\mathrm{R}_{1}$ has $\left.0 \epsilon, \mathrm{R}_{2} 3 \epsilon\right)$, one actually obtains

$$
\frac{(\mathrm{N}-1+\mathrm{P}) !}{(\mathrm{N}-1) ! \mathrm{P} !}=\frac{(2-1+3) !}{(2-1) ! 3 !}=4
$$

different distributions of the resonators $R_{1}, R_{2}$ over the energy-grades. 
We may summarize the above as follows:-Einstein's hypothesis leads necessarily to formula $(\alpha)$ for the entropy and thus necessarily to Wien's radiation-formula, not Planck's. Planck's formal device (distribution of P energyelements $\epsilon$ over $\mathbf{N}$ resonators) cannot be interpreted in the sense of Einstein's light-quanta.

\section{The Visibility of Radiation.} By P. G. Nul'isG*.

$\checkmark \mathrm{HE}$ quantitative relation between light and radiation has long been sought by many investigators. Herschel, exploring the spectrum with a thermometer, found that the radiation continued beyond what was visible. The invisible ultra-violet portions of spectra were long ago explored by photography. Langley $\dagger$, twenty-five years ago, explored the infra-red solar spectrum with his fine wire bolometer, and in the visible spectrum measured the amounts of energy of various wave-lengths required for reading print. Pflüger $\ddagger$ and König and Dieterici $\$$ determined the relative amounts of energy required to just produce a luminous sensation in different parts of the spectrum. König $\|$ continued his investigations from the threshold of vision up to an intensity of about 500 metre candles.

About ten years ago it was clearly recognized that in order to define light in terms of the radiation which excites it, an intermediate function, the visibility of radiation, must be formulated and its constants determined for the average normal eye. Goldhammer 7 , in 1905 , partly reduced some of König's data and expressed visibility as a function similar in form to that giving the spectral energy of a perfect radiator. Hertzsprung ${ }^{*}$, in 1906 , took a rough average of all available threshold data and formulated visibility as a logarithmic hyperbola. The author $+\dagger$, independently of Goldhammer and Hertzsprung, reduced the data of Langley, Pflüger, and König, and in 1907 published this, a function

* Communicated by the Author.

† S. P. Langley, Am. Journ. Sci. xxxvi. p. 359 (1886).

\pm A. Pflüger, Ann. Ph. ix. p. 185 (1902).

$\$$ König and Dieterici, Zs. Psy. Phys. Sinn. iv. p. 241 (1893),

\| A. König, Ges. Abhandlungen.

ๆ I). A. Goldhammer, Ann. Ph. xvi. p. 621 (1905).

** E. Hertzsprung, Z. Wiss. Phot. iv. p. 43 (1906).

t† P. G. Nutting, Phys. Rev. xxiv. p. 202 (1907); Bull. Bu. Stds. v. p. 261 (1908). 